\title{
Intracranial EEG Connectivity Analysis and Result Imaging
}

\author{
Petr Klimeš, Jiř́ Janeček, Pavel Jurák, Josef Halámek, Jan Chládek, and Milan Brázdil
}

\begin{abstract}
We are presenting a method for analyzing signals from deep brain structures, measured by intracranial electrodes, to reveal connectivity in the human brain. Time evaluation of the correlation technique is applied for every available contact pair to determine dependencies between EEG channels. This produces a large amount of results that are not easy to interpret. Here we introduce a procedure for complex and comprehensive result imaging which helps us focus on significant parts of the results. Changes of power are examined along with correlation changes. Results are demonstrated for one subject. Five channels were selected from the hippocampus for detailed analysis. How the changes of correlation are connected to changes of power is shown in addition to the coupling between channels.
\end{abstract}

Index Terms-Connectivity, correlation, intracranial EEG, signal processing.

\section{INTRODUCTION}

Individual neuronal populations in the human brain co-operate with each other during various tasks using mild electric signals in various frequency bands [1]. These measurable signals reflect the real physiological activity in the brain. Therefore, one of the greatest challenges is defined as finding the best way to read this information hidden in various phenomena and determining how the brain structures are connected and how the signals are spread.

Generally speaking, there are a couple of approaches for perceiving neuronal networks according to the level of spatial range. Basically, we can examine human brain structures on three levels. The macroscopic level among large neuronal networks, which can be measured by ordinary scalp EEG, the mesoscopic level reaching individual neuronal populations and their field potential oscillations, and the microscopic level considering well-described single neuron oscillations [2].

Our data from depth electrodes accommodates information with good time and spatial resolution about the signals generated and carried by internal structures. Unlike scalp EEG, which can only provide us with a summarization of these local signals [3], intracranial measurement allows us to achieve an extremely detailed insight into internal structures

Manuscript received June 22, 2012; revised July 30, 2012. This study was supported by the projects: GACR P103/11/0933, ALISI CZ.1.05/2.1.00/01.0017 and CEITEC CZ.1.05/1.1.00/02.0068 from European Regional Development Fund.

Petr Klimeš, Jiří Janeček, Pavel Jurák, Josef Halámek, and Jan Chládek are with the Institute of Scientific Instruments, Academy of Sciences of the Czech Republic, Brno, Czech Republic.

Milan Brázdil was with the Behavioural and Social Neuroscience Research Group, CEITEC - Central European Institute of Technology, Masaryk University, Brno, Czech Republic. Now he is with the Brno Epilepsy Center, Department of Neurology, St. Anne's University Hospital and Medical Faculty of Masaryk University, Brno, Czech Republic. and reveal their functional habits at the level of individual neuronal populations. Focusing on specific areas in the brain, we can study signal amplitudes in various frequency bands, as well as dependencies between two signals from different contacts.

It is important to understand what kind of signal is being processed. In the case of an EEG signal, its stochastic and non-stationary character with a wide frequency range must also be considered, in addition to its very low amplitude. To reveal similarities between two such complex signals, correlation with its relatively simple mechanism can be extremely effective. Many methods for revealing connectivity between EEG channels have been presented in the literature. Correlation, however, represents the basis for the majority of them. The group of specialized methods based on multivariate autoregressive models (MVAR), such as asymmetric Directed Transfer Function or Partial Directed Coherence, is extremely-powerful [4], [5]. These sophisticated methods, capable of distinguishinh connectivity together with flow direction, are, however, not successfully applicable to data from isolated regions recorded by intracerebral electrodes. MVAR methods are supposed to work with a complex dataset usually captured by scalp EEG [6]. It is not common to work with a predefined dataset in intracranial EEG recordings. Single contacts are very often included or excluded from a set. Therefore, the above-mentioned correlation, together with certain statistical tests, was chosen for intracranial EEG processing.

Analyzing signals, and especially the connectivity between them, produces a large number of results. For a subject with 90 recorded EEG contacts, more than 4,000 couples are produced which can be observed as an ensemble and which inform us only about activity and interactions over all channels (Fig. 1). The resuls must be reduced to achieve a better orientation. This presents another challenge - how to process these results without missing any important parts.

The following parameters must be considered during intracranial EEG processing: frequency, type of signal (time signal or its power envelope), and the time-space distribution of the signal in the brain. An ideal imaging method should, therefore, provide us with a well-arranged overview over a whole set of results along with the option of focusing our attention more closely on specific parts of the results. As mentioned, this focus should consider different frequency ranges for time signals or their power envelopes in addition to the time axis. To focus on spatial resolution, the imaging method should be able to select and adjust results for certain groups of contacts without losing information about their real position in the brain and their relations to other contacts in their surroundings.

The method presented in this paper follows previously published methods which provide us with an overview of 
thousands of EEG channel pairs [7]. New imaging methods allow us to focus on selected areas in the brain and perform additional analysis on these structures.

In the following chapters the procedure of intracranial EEG recordings is briefly described. An insight into our signal processing methods is then presented, and results for one selected subject are shown before the conclusion.

\section{METHODS}

\section{A. EEG Recordings}

The data presented here are used only to demonstrate the methodology of signal processing. There is no neurological conclusion in this paper, for which reason only basic information about the patients is given. Patients with pharmacoresistant epilepsy participated in the study. Standard semiflexible intracerebral electrodes were implanted in order to localize seizure origin before neurosurgical treatment. Electrodes were placed so as to reach specific areas, e.g. the amygdala, hippocampus, etc. The exact position of electrodes in the brain was verified using post-placement MRI. All recordings were monopolar with earlobe reference. The number of channels for each subject ranged from 87 to 111 in the example presented. The sampling rate was $1,024 \mathrm{~Hz}$. Anatomical structures with any kind of disorder were omitted from the analysis.

A visual oddball task was performed to record standard repetitive EEG [1]. Two types of simple stimuli were used frequent and target. Subjects were requested to ignore frequent stimuli and to perform a certain task (motor response, counting, etc.) when target stimuli appeared. The interval between stimuli varied randomly ( $4-6 \mathrm{~s})$ and the duration of stimulus exposure was $500 \mathrm{~ms}$. The target stimulus was supposed to activate whole sets of cognitive processes in the brain. These processes are mostly linked to attentional mechanisms, short term memory, assessment of stimulus relevance, decision-making, and response [8].

\section{B. Signal Processing}

\section{1) Pre-processing}

The measured data are processed off-line. Channels with corrupted information are excluded and the whole remaining data are segmented before analyzing. Segmentation is performed for every channel in accordance with the stimuli interval. The length of the segment is usually 4 or $8 \mathrm{~s}$ and the onset of stimulation is always placed in the middle of each segment. Thereafter segments with artifacts are omitted from selection.

To suppress common far fields the bipolar montages of selected contacts are calculated as the difference between two neighboring contacts and the difference between the first and last contact on one multipolar electrode.

Segmented and artifact-free channels, along with their bipolar montages, can be further processed as responses to target or frequent stimulation. This means that segments from each channel are divided into target and frequent segments and analyzed separately.

In the next step, every sequence of segments can be filtered as time signals or power envelopes in different frequency ranges. These frequency ranges usually cover frequencies from $2 \mathrm{~Hz}$ to $90 \mathrm{~Hz}$. Different band-widths can be used.

\section{2) Coupling}

At this point, segmented and filtered signals are prepared for analysis. Time evaluation of correlation is applied to determine the coupling between EEG channels. Two signals from different channels are compared to each other through floating windows moving over each segment of both signals. Pearson's correlation coefficient is calculated for every window step according to expression (1) and the result, which yields in a range from -1 to 1 , is placed in the middle of the floating window. Both selected windows are multiplied by Hamming window prior to correlation coefficient calculation to decrease the influence of border parts.

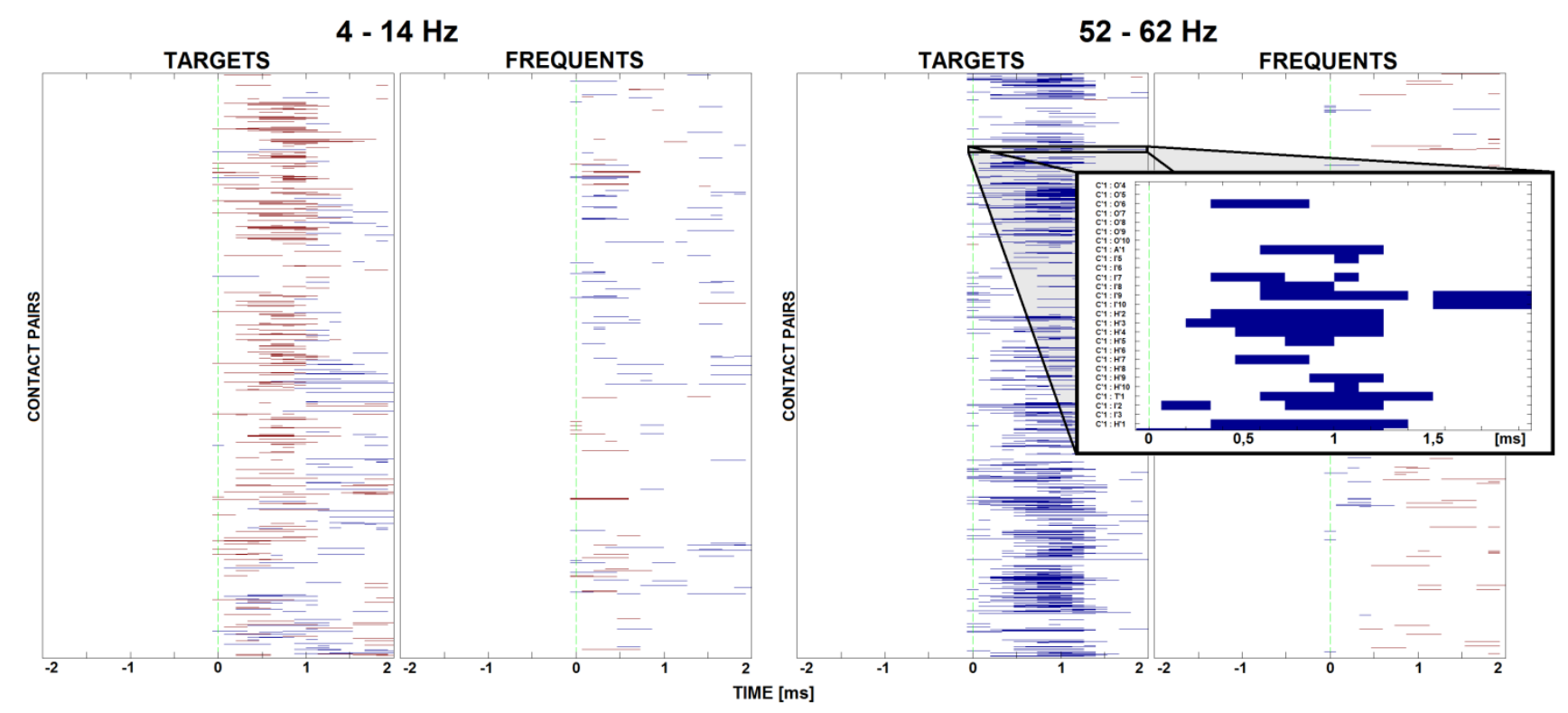

Fig. 1. Subject 39. Correlation of time signals in $4-14 \mathrm{~Hz}$ and $52-62 \mathrm{~Hz}$. Probability value p < 0.01. All contact pairs (over 4,000), every individual one represented by one row. The position of the stimulus is at $0 \mathrm{~ms}$. Statistically significant changes (red - increases, blue - decreases) for targets and frequents. 
The essential parameter for correlation calculation is the width of the floating window and its step. A shorter window can achieve a better time resolution, though it is important to consider the character of the signal and what the purpose of our analysis is. Too short a window can produce false results by calculating the correlation between pieces of signals that are too short while too long a window can average results too much. The step of the window should be set in accordance with its width (usually $10 \%$ of window width). Both width and step, are crucial to the final shape of the results.

$$
\rho_{x y}(m, n)=\frac{C_{x y}(m, n)}{\sqrt{\sigma_{x}^{2}(m) \sigma_{y}^{2}(n)}}
$$

The correlation function for every available contact pair is created from the sequence of correlation coefficients. The number of points of this correlation function is dependent on segment length, floating window width, and its step. Correlation functions from all segments are then averaged and this average correlation function, presenting the average correlation between a certain pair of channels, is used for further analysis. This procedure is performed for all contact pairs.

In addition to the level of correlation, it is also important to find significant parts of its changes, which reflect activity bound to the stimuli. Changes of correlation are analyzed by comparing levels of correlation before and after stimulation. A baseline $(B L)$ is set in every correlation function, which is supposed to represent relaxed activity. In the case of a $4 \mathrm{~s}$ segment length, $B L$ started $600 \mathrm{~ms}$ before stimulation and ended $100 \mathrm{~ms}$ before stimulation. The section after stimulation is then examined part-by-part using a different floating window, one-third the width of the $B L$. The mean value of correlation from every step of the window is compared to the mean value from $B L$.

A probability value $(p)$ using a non-parametric Wilcoxon Rank Sum test for paired samples is applied to distinguish any statistically significant difference between BL and the interval demarcated by the floating window. The difference is marked as significant for $p<0.01$. The change of correlation after stimulus is considered as an increase (synchronization) if the statistically significant value from the floating window is higher than the mean value from BL, and in the case of lower values the change is considered a decrease (desynchronization).

\section{3) Power changes}

Examination of power and its significant changes in every channel can inform us how the synchronization or desynchronization in correlation is connected to power increases $(E R S)$ and decreases $(E R D)$. This is again done by setting the BL before stimulus and comparing the mean value of power from this interval to the mean value of power from the floating interval $(F I)$ demarcated by the window, which in this case is again floating over the whole segment. Normalization with BL is performed to highlight changes in power changes. When the mean value from FI is greater than the mean value from $B L(E R S)$, the resulting value of power is normalized according to equation (2). When the value from $F I$ is smaller than the value from $\mathrm{BL}(E R D)$, the resulting value of power is normalized according to equation (3).

$$
\begin{aligned}
& E R S=\left(\frac{F I}{B L}-1\right) \times 100 \\
& E R D=\frac{1}{\frac{F I}{B L}-1} \times 100
\end{aligned}
$$

The limitation $+/-100$ of normalized values is used and the resulting scale is $+100,-100$. Value +100 , represented in the result matrix by a dark red color, means a doubling of instantaneous power with respect to BL. Value -100 , represented by a dark blue color, means a drop of one half. An example can be seen in Fig. 5.

To demonstrate correlation and power computation for selected data in this example, approximately 40 target and 40 frequent segments from each channel were separately investigated in the $4-14 \mathrm{~Hz}$ and $52-62 \mathrm{~Hz}$ frequency ranges filtered as time signals. Bipolar montages were calculated for selected channels. Correlation was calculated for these channels by a floating window $1 \mathrm{~s}$ in width with a $0.1 \mathrm{~s}$ step. A baseline $0.5 \mathrm{~s}$ wide with a floating window one-third the width of baseline was used for calculation of significant changes in correlation and power. No time delay between channels was considered.

\section{Imaging}

It is not easy to choose an appropriate form in which to display the results. Fig. 1 demonstrates a method of general graphical visualization of information about interactions over all channels. Significant increases and decreases of correlation after stimulation are drawn as red and blue points in a matrix, where each row represents the correlation development in time between one pair of contacts. Fig. 1 shows an example of this projection for targets and frequents in the $4-14 \mathrm{~Hz}$ and $52-62 \mathrm{~Hz}$ frequency ranges.

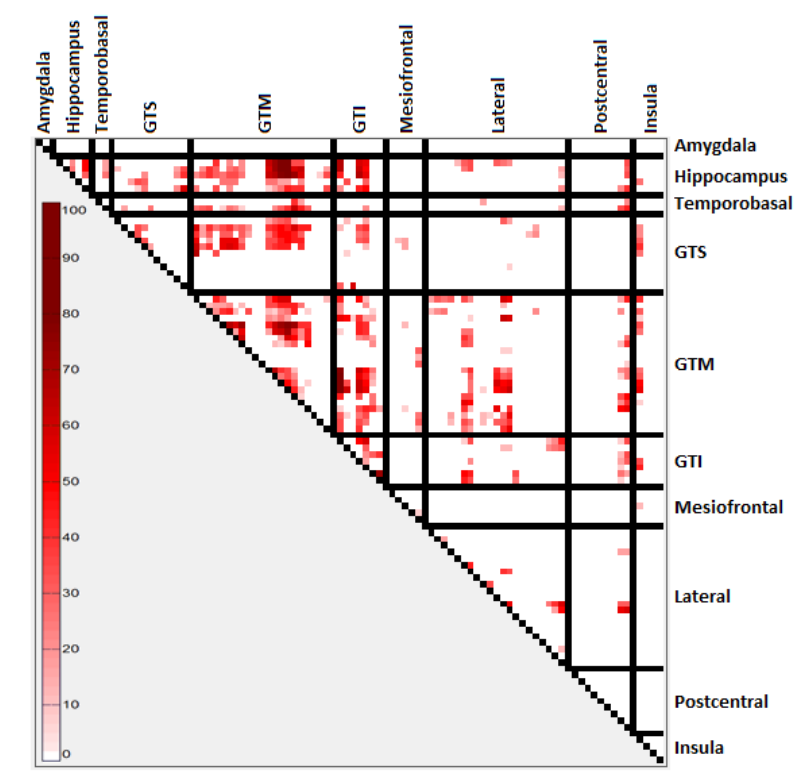

Fig. 2. Example of spatial projection of all channels showing a level of correlation in particular areas. Every point in the matrix represents one correlation pair - one row from the matrices in Fig. 1. The matrix is symmetrical, for which reason only one half needs to be shown.

As we can see the majority of activity takes place approximately $500 \mathrm{~ms}$ after stimuli. This focuses our interest on a precise time interval. However, it is impossible to 
observe individual structures or even single channels by this kind of imaging without removing them from the context of other signals. A different type of result interpretation must be employed to obtain information about spatial relations.

Fig. 2 shows the same data as Fig. 1 with individual contact pairs grouped by their real placement in the brain. On the basis of previous projection, only the data from interval $250-750 \mathrm{~ms}$ after stimuli were used. The tone of the color provides information about the amount of significant points in the selected interval $(250-750 \mathrm{~ms}$ after stimuli). This clearly shows which structures have the greatest significant changes in correlation and allows us to focus our attention on these areas.

From this point each area can be inspected separately. This brings us even deeper into the examination and with substantially reduced data it is now possible to perform further analysis and observe how single channels in chosen areas react to stimulus and interact with each other. Fig. 3 shows specific results for target increases and decreases in selected frequency ranges. Significant increases are detected in low frequency ranges and remarkable decreases at high frequencies.

The contacts from the hippocampus were selected for deeper analysis in our case. The circle in Fig. 3 shows the given area. Strong synchronization can be seen between contacts B'1: C'2 as well as between contacts B'2: C'2. Significant synchronization is also detected between B'3: C'2 and B'3: B'2.

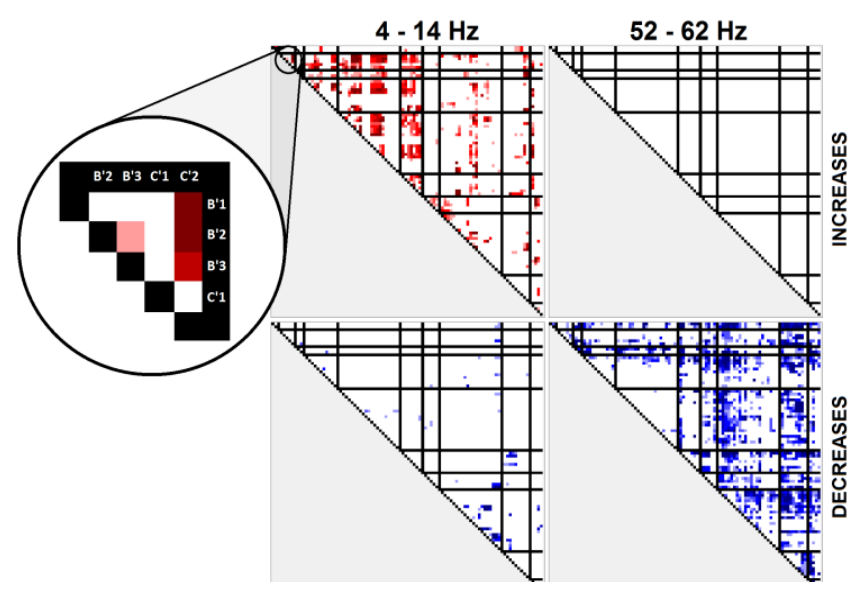

Fig. 3. Subject 39. Correlation of time signals in $4-14 \mathrm{~Hz}$ and $52-62 \mathrm{~Hz}$. Probability value $\mathrm{p}<0.01$. Interval $250-750 \mathrm{~ms}$ after stimulation. The zoomed area shows how contacts placed in the hippocampus interact with each other.

\section{RESULTS}

Having a reduced number of channels selected from specific structures, the correlation can be calculated more precisely along with the power levels of every channel. Five channels (B'1, B'2, B'3, C'1, C'2) from an area of the hippocampus and their bipolar montages weve selected for upcoming presentation. Fig. 4 shows the results of correlation in three matrices for two selected frequency ranges.
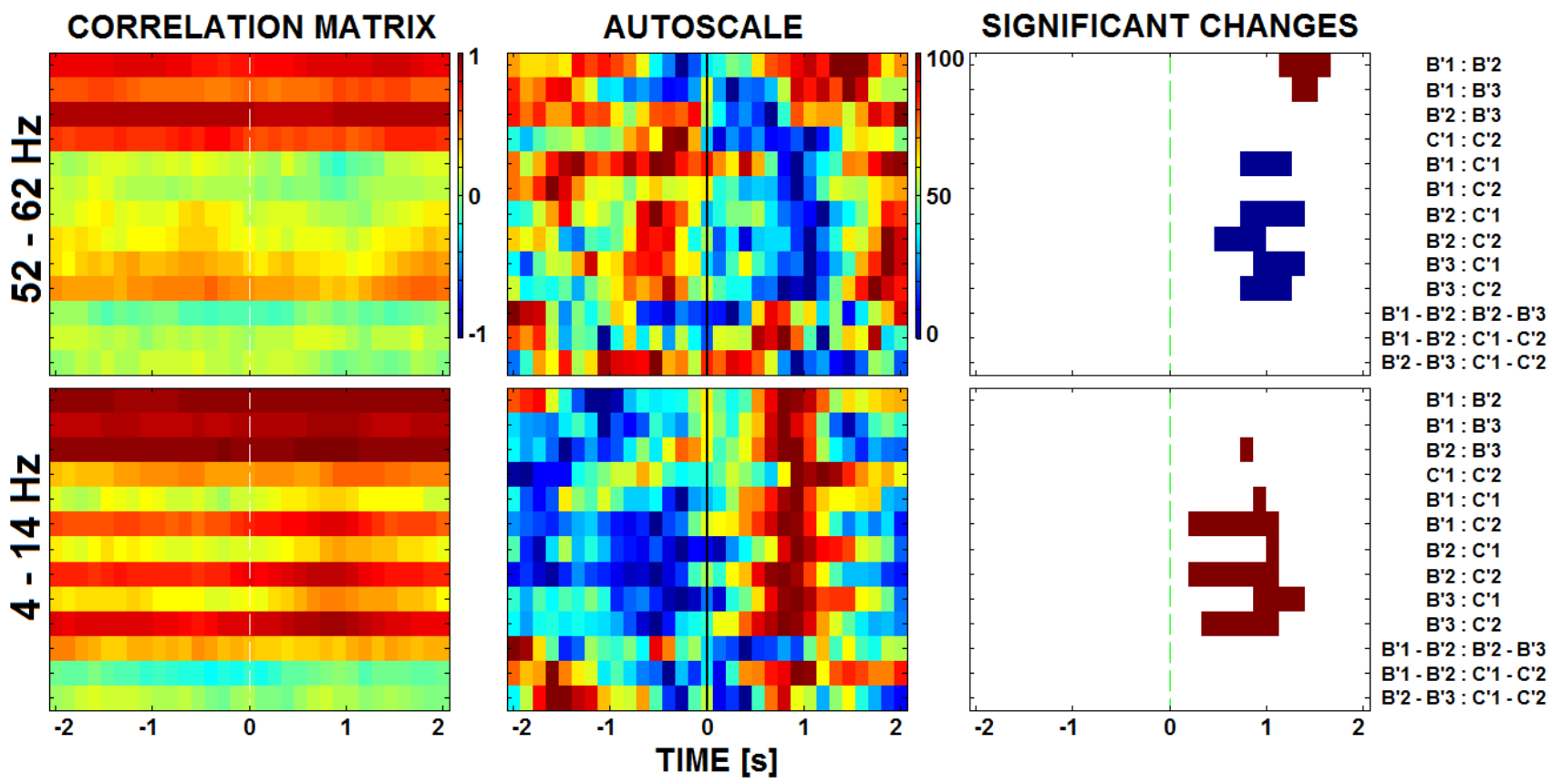

Fig. 4. Subject 39. Correlation of time signals in $4-14 \mathrm{~Hz}$ and $52-62 \mathrm{~Hz}$. Correlation matrices for selected channels and their bipolar montages. The first ten rows from the top in every matrix represent correlation between selected unipolar channels (B'1, B'2, B'3, C'1, C'2). The remaining three rows represent correlation for bipolar montages. Stimulus occurs at $0 \mathrm{~s}$. LEFT - Correlation matrix shows the values of correlation coefficients in range -1, 1. MIDDLE Autoscaled correlation matrix highlights changes in correlations by autoscaling each row - maximum value is equal to $100 \%$ and minimum to $0 \%$. RIGHT Significant changes of correlation between the mean value of the baseline and the mean values in floating window. Three levels - increases (red), decreases (blue), and no significant change (white). Probability value $\mathrm{p}<0.01$. 

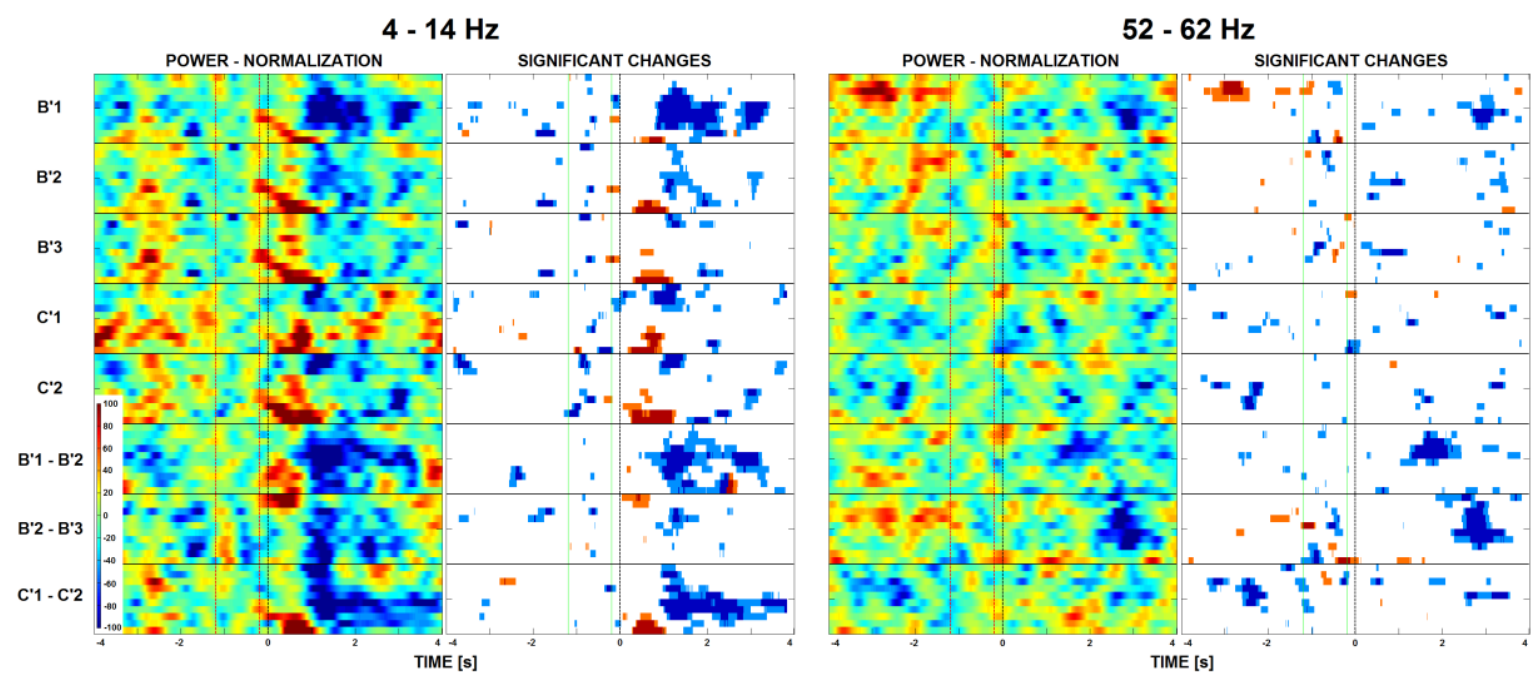

Fig. 5. Subject 39. Normalization of power envelopes and significant changes at $4-14 \mathrm{~Hz}$ and $52-62 \mathrm{~Hz}$. Power normalization is performed according to equations (2) and (3). The significant changes of power levels are based on the difference between the mean value of power at the baseline (marked in significant changes by green lines) and the floating window. The power levels for each channel are divided into ten additional rows. Each row represents one hertz step in the selected frequency range (from bottom to top), with a $4 \mathrm{~Hz}$ frequency band. Stimulus occurs at $0 \mathrm{~s}$.

The results show high correlation between channels from the same electrode (B and C). This is caused by parasitic signals which disseminate along the electrode. At a low frequency, there is also a high correlation between channels B'1 : C'2, B'2 : C'2, and B'3 : C'2, which corresponds with the results in Fig. 3. Significant increases of correlation after stimulation (synchronization) were detected at low frequencies and significant decreases (desynchronization) in high frequency range.

The power levels in Fig. 5 show that all contacts have similar reactions on stimulation in the designated frequency ranges. Decreases (ERD) with slight delay are detected at low frequencies. No significant changes are detected at higher frequencies.

When these results are compared with correlations, a significant increase in correlation at low frequencies seems to be bound to ERD. On the other hand, desynchronization (correlation decrease) at high frequencies is not accompanied by any significant changes of power.

In any case, our results imply that changes of correlation are very often accompanied by significant changes in power of an opposite manner - an increase of correlation with a decrease of power at low frequencies and a decrease in correlation with an increase of power at higher frequencies.

\section{DISCUSSION AND CONCLUSION}

Large-scale neural networks are investigated most commonly in human brain studies. It is impossible to reveal the details of information processing in brain dynamics from this macroscopic view. The mesoscopic scale, able to obtain more precise data from particular structures in the human brain, can be achieved by using intracerebral macro- or micro-electrodes. Recordings of intracranial EEG produce a large amount of data. Investigation of more than 4,000 combinations must be performed to evaluate coupling between 90 channels. This requires special methods that take us through the data and focus our attention on the important parts.

The set of methods presented in this paper is able to process thousands of EEG signals in a data set and reveal structural relations from the resulting matrices by progressive focusing through time and spatial dimensions.

The results are easy to interpret, though interpretation must be performed with respect to the character of the data and the parameters of the calculations. The position and width of the baseline can have a crucial influence on the results, in addition to the width of the floating window and its step. Another important fact is that no time delay between channels was considered. This means that this method assumes reaction to stimulation in two places at the same time.

Progress in future work should attempt to achieve more detailed results along with time delay investigation between signals. Correlation of shifted signals can disclose how the signals are really spread in the human brain. However, this approach is questionable, because it works on the assumption that signals keep their shape as they spread.

\section{REFERENCES}

[1] M. Brazdil, et al., "On the time course of synchronization patterns of neuronal discharges in the human brain during cognitive tasks," in Frontiers in Human Neuroscience, submited for publication.

[2] C. K. Young and J. J. Eggermont, "Coupling of mesoscopic brain oscillations: recent advances in analytical and theoretical perspectives," in Progress in Neurobiology, vol. 89, pp. 61-78, 2009.

[3] G. Gomez-Herrero, M. Atienza, K. Egiazarian, and J. L. Cantero, "Measuring directional coupling between EEG sources," in NeuroImage, vol. 43, pp. 497-508, 2008.

[4] P. Jiruska, J. Proks, O. Drbal, P. Sovka, P. Marusic, and P. Mares, "Comparison of different methods of time shift measurement in EEG", in Physiological Research, vol. 54, no. 4, pp. 459-465, 2005.

[5] A. Schoegl and G. Supp, "Analysing event-related EEG data with multivariate autoregressive parameters," in Progress in Brain Research, vol. 159, pp. 135-145, 2006.

[6] L. Baccala and K. Sameshima, "Overcoming the limitations of correlation analysis for many simultaneously processed neural structures," in Progress in Brain Research, vol. 130, pp. 33-47, 2000.

[7] J. Janecek, J. Chladek, J. Halamek, P. Jurak, and M. Brazdil, "Analysis of time evolution of couplings in the repetitive EEG," in ACTA Press. [Online].

http://www.actapress.com/Abstract.aspx?paperId=453208

[8] M. Brazdil, et al., "Directional functional coupling of cerebral rhythmsbetween anterior cingulate and dorsolateral prefrontal areas during rare stimuli: A directed transfer function analysis of human depth eeg signal," in Human Brain Mapping, vol. 18, no. 4, pp. 138-146, 2009. 